International Mathematical Forum, 2, 2007, no. 50, 2491 - 2502

\title{
2-Local Isometries and 2-Local Automorphisms on Uniform Algebras ${ }^{1}$
}

\author{
Osamu Hatori \\ Department of Mathematics, Faculty of Science \\ Niigata University, Niigata 950-2181 Japan \\ hatori@math.sc.niigata-u.ac.jp
}

Takeshi Miura

Department of Applied Mathematics and Physics

Graduate School of Science and Engineering

Yamagata University, Yonezawa 992-8510, Japan

miura@yz.yamagata-u.ac.jp

Hirokazu Oka

Faculty of Engineering, Ibaraki University

Hitachi 316-8511 Japan

oka@mx.ibaraki.ac.jp

Hiroyuki Takagi

Department of Mathematical Sciences

Faculty of Science, Shinshu University

Matsumoto 390-8621 Japan

takagi@math.shinshu-u.ac.jp

\begin{abstract}
In this paper we study 2-local isometries and 2-local automorphisms on uniform algebras. In particular, we show that 2-local isometries are surjective linear isometries for certain algebras of holomorphic functions of one and two complex variables.
\end{abstract}

\footnotetext{
${ }^{1}$ The authors were partly supported by the Grants-in-Aid for Scientific Research, The Ministry of Education, Science, Sports and Culture, Japan.
} 


\section{INTRODUCTION}

Let $\mathcal{A}$ be a complex linear space and $\mathcal{L}(\mathcal{A})$ the algebra of all linear operators on $\mathcal{A}$. Suppose that $S$ is a subset of $\mathcal{L}(\mathcal{A})$. A linear operator $\mathcal{T}$ on $\mathcal{A}$ is said to be locally in $S$ if for each $a \in \mathcal{A}$ there is $\mathcal{T}_{a} \in S$ with $\mathcal{T}(a)=\mathcal{T}_{a}(a)$. We say that $S$ is algebraically reflexive, if every linear operator which is locally in $S$ belongs to $S$. For the case where $\mathcal{A}$ is an algebra a linear operator $\mathcal{T}$ on the algebra $\mathcal{A}$ is said to be a local automorphism if $\mathcal{T}$ is locally in the group of all (algebra) automorphisms on $\mathcal{A}$. In this paper, an automorphism is always an algebra automorphism. For the case where $\mathcal{A}$ is a Banach space a linear operator $\mathcal{T}$ on the space $\mathcal{A}$ is said to be a locally surjective isometry if $\mathcal{T}$ is locally in the group of all surjective linear isometries on $\mathcal{A}$. Reflexivity problems for Banach algebras was initiated by Kadison and Larson. Kadison [6] studied the reflexivity of the algebras of all derivations on von Neumann algebras. Larson [8] posed the question of the reflexivity of the automorphism groups of Banach algebras. These problems are also investigated for several function spaces in $[5,11,12,13,16,17,18]$ and others.

Molnár and Zalar [13, Theorem 2.2] proved that the group of all surjective linear isometries of $C(X)$ are algebraically reflexive if $X$ is a first countable compact Hausdorff space. It is not the case unless $X$ is first countable. In fact, Sánchez and Molnár [18, Theorem 9] showed it by proving that every unital injective endomorphism of $C(\beta \mathbb{N} \backslash \mathbb{N})$ is a local automorphism, where $\mathbb{N}$ is the discrete space of all the positive integers and $\beta \mathbb{N}$ is its Stone-Čech compactification. On the other hand Sánchez [17] proved that for a $\sigma$-finite measure $\mu$, the group of all automorphisms of $L_{\infty}(\mu)$ is algebraically reflexive if and only if $L_{\infty}(\mu)$ is $*$-isomorphic to either $\ell_{\infty}(\Gamma)$ or $\ell_{\infty}(\Gamma) \times L_{\infty}([0,1])$, where $\Gamma$ is a countable set. Sánchez and Molnár [18] also investigated the same problem for the algebra of analytic functions. Let $K$ be a compact subset of the complex plane $\mathbb{C}$ whose complement has finitely many components. Suppose that $A(K)$ is the algebra of all complex-valued continuous functions on $K$ which are analytic on the interior of $K$. Sánchez and Molnár [18, p.423] showed that the group of all surjective linear isometries of $A(K)$ is algebraically reflexive. We will show that it is the case for every compact subset $K$ of the complex plane whose interior of $K$ is connected and the closure of the interior of $K$ is $K$.

On the other hand unless we assume linearity on the maps, non-linear local automorphisms and non-linear locally surjective isometries can be almost arbitrary. Instead of linearity we assume 2-locality for the maps which was introduced by Šemerl [19] motivated by the paper of Kowalski and Słodokowski [7]. Let $T$ be a map from a Banach algebra $A$ into itself. No continuity, surjectivity, linearity nor multiplicativity are assumed here. $T$ is called a 2-local 
automorphism (resp. 2-local isometry) if for any $f, g \in A$, there exists an automorphism (resp. surjective linear isometry) $T_{f, g}$ on $A$ with $T(f)=T_{f, g}(f)$ and $T(g)=T_{f, g}(g)$. Šemerl first studied 2-local automorphisms together with other things in [19] and he proved that every 2-local automorphism on a Banach algebra $B(H)$ of all bounded linear operators on an infinite dimensional separable Hilbert space $H$ is an automorphism. Molnár [10] initiated 2-local isometries and proved that for certain $C^{*}$-subalgebras of $B(H)$, 2-local isometries are surjective linear isometries. For the case of commutative Banach algebras, Molnár [11, p.119] showed that every 2-local automorphism on $C(\mathcal{X})$ for a first countable compact Hausdorff space $\mathcal{X}$ is an automorphism, where $C(\mathcal{X})$ denotes the algebra of all complex-valued continuous functions on $\mathcal{X}$. Györy [4] proved that every 2-local isometries on $C_{0}(\mathcal{Y})$ is a surjective linear isometry if $\mathcal{Y}$ is a first countable and $\sigma$-compact locally compact Hausdorff space, where $C_{0}(\mathcal{Y})$ is the algebra of all complex-valued continuous functions which vanish at infinity on $\mathcal{Y}$. Györy also showed in the same paper that for an uncountable discrete space $L$, there exists a non-sujective 2-local automorphism on $C_{0}(L)$.

In this paper we study 2-local automorphisms and 2-local isometries on uniform algebras including certain algebras of holomorphic functions of one and two complex variables. We say that $A$ is a uniform algebra on a compact Hausdorff space $X$ if $A$ is a closed subalgebra of $C(X)$ which separates the points of $X$ and contains constant functions. For a subset $K$ of $X$, the supremum norm on $K$ is denoted by $\|\cdot\|_{\infty(K)}$. We denote the spectrum of $f \in A$ by $\sigma(f)$. Note that a 2-local automorphism on a uniform algebra is a 2-local isometry since every automorphism on a uniform algerba is a surjective linear isometry.

\section{2-LOCAL AUTOMORPHISMS}

Let $A$ be a uniform algebra on a compact Hausdorff space $X$. It is well-known that an automorphism on $A$ is represented by the composition operator; for an automorphism $T$ on $A$ there corresponds a homeomorphism $\varphi$ from the maximal ideal space $M_{A}$ of $A$ onto itself such taht $\widehat{T(f)}=\hat{f} \circ \varphi$ on $M_{A}$ for every $f \in A$, where $\hat{\imath}$ denotes the Gelfand transformation. In particular, every automorphism on a uniform algebra is a surjective linear isometry.

Lemma 2.1. Suppose that $A$ is a uniform algebra on a compact Hausdorff space $X$. If the group of all surjective isometry on $A$ is reflexive, then the group of all automorphism is reflexive.

Proof. Suppose that $S$ is a local automorphism on $A$. Since automorphisms are surjective isometries on uniform algebras, we have that $S$ is a locally surjective isometry. So $S$ is a surjective linear isometry by the hypothesis. Then by a theorem of de Leeuw, Rudin and Wermer [3, Theorem 3] there are a $\tau \in A$ with $\tau(X) \subset\{|z|=1\}$ and an automorphism $T$ on $A$ such that $S(f)=\tau T(f)$ 
( $f \in A$ ). On the other hand $S(1)=1$ holds since $S$ is a local automorphism. It follows that $\tau=1$ and so $T=S$. Thus $S$ is an automorphism on $A$.

Molnár [11, p.119] showed that any 2-local automorphism on $C(X)$ for a first countable compact Hausdorff space $X$ is an automorphism. To prove this Molnár first showed that $T$ is linear by applying [7]; $T$ is a local automorphism. Since the group of all surjective linear isometries on $C(X)$ is reflexive if $X$ is first countable [13], $T$ is an automorphism by Lemma 2.1. This can be formally generalized in the completely same argument for uniform algebras. Since we will apply the result later in this paper we show it with a proof for a convenience.

Theorem 2.2. Let $A$ be a uniform algebra and $T$ a 2-local automorphism on $A$. Then $T$ is an isometrical isomorphism from $A$ onto $T(A)$. In particular, if the group of all automorphisms on $A$ is algebraically reflexive, then every 2-local automorphism is an automorphism.

Proof. Let $x \in M_{A}$, the maximal ideal space of $A$. Put $S_{x}: A \rightarrow \mathbb{C}$ by $S_{x}(f)=\widehat{T(f)}(x)$ for every $f \in A$, where $\widehat{\cdot}$ denotes the Gelfand transformation. Suppose that $f, g \in A$. Then there is an automorphism $T_{f, g}$ on $A$ such that $T(f)=T_{f, g}(f)$ and $T(g)=T_{f, g}(g)$, so

$$
\left.S_{x}(f)-S_{x}(g)=T_{f, g} \widehat{(f-g}\right)(x) \in \sigma\left(T_{f, g}(f-g)\right)=\sigma(f-g)
$$

holds. Putting $f=0$ and $g=1$ we have $T(0)=T_{0,1}(0)=0$ for an automorphism $T_{0,1}$, so $S_{x}(0)=0$. It follows that $S_{x}$ is linear and multiplicative by a theorem of Kowalski and Słodkowski [7]. Thus $S_{x}$ is a non-zero complex homomorphism for $S_{x}(1)=\widehat{T_{0,1}(1)}=1$; we may suppose that $S_{x} \in M_{A}$. Put a function $\varphi: M_{A} \rightarrow M_{A}$ defined by $\varphi(x)=S_{x}$ for $x \in M_{A}$. Then $\hat{f}(\varphi(x))=\widehat{T(f)}(x)$ holds for every $f \in A$ and $x \in M_{A}$, so that $T$ is an endomorphism on $A$ since $A$ is semi-simple. So, by definition, $T$ is a local automorphism. For every $f \in A$ there is an automorphism $T_{f}$ on $A$ such that $T(f)=T_{f}(f)$. Then there is a homeomorphism $\varphi_{f}$ on $M_{A}$ such that $\widehat{T_{f}(f)}=\hat{f} \circ \varphi_{f}$. Thus

$$
\begin{aligned}
\sigma(f)=\hat{f}\left(M_{A}\right)=\hat{f} \circ \varphi_{f}\left(M_{A}\right) & \\
& =\widehat{T_{f}(f)}\left(M_{A}\right)=\widehat{T(f)}\left(M_{A}\right)=\sigma(T(f)) .
\end{aligned}
$$

It follows that $T$ is an isometrical isomorphism from $A$ onto $T(A)$. If the group of all automorphisms on $A$ is reflexive, then $T$ is an automorphism.

In a way similar to Györy [4] we show that there are a compact Hausdorff space $\overline{\mathcal{K}}$ which is not first countable and a 2-local automorphism on $C(\overline{\mathcal{K}})$ which is not surjective. The idea of constructing such an operator is also essentially the same as that of Examples 1 and 2 of Sánchez and Molnár [18]. 
Let $\kappa$ be an infinite cardinal number. Let $\mathcal{K}$ be a discrete space with the cardinality $\nu$ which is strictly larger than $\kappa$. Note that $\nu$ is strictly larger than the countable cardinal number. The one point compactification of $\mathcal{K}$ is denoted by $\overline{\mathcal{K}}=\mathcal{K} \cup\{\infty\}$. Then $\overline{\mathcal{K}}$ is not first countable since $\mathcal{K}$ is not countable. Choose a countable subset $\left\{x_{n}\right\}_{n=0}^{\infty}$ of $\mathcal{K}$ and put $\mathcal{L}=\mathcal{K} \backslash\left\{x_{n}\right\}_{n=0}^{\infty}$. Put a function $\varphi_{0}$ from $\overline{\mathcal{K}}$ onto $\overline{\mathcal{K}}$ by $\varphi_{0}\left(x_{0}\right)=\varphi_{0}(\infty)=\infty, \varphi_{0}\left(x_{n}\right)=x_{n-1}$ for every $n \geq 1, \varphi_{0}(y)=y$ for every $y \in \mathcal{L}$. Then $\varphi_{0}$ is continuous. Put a composition operator $T_{0}$ on $C(\overline{\mathcal{K}})$ by $T_{0}(f)=f \circ \varphi_{0}$ for $f \in C(\overline{\mathcal{K}})$. Then $T_{0}$ is an isometric isomorphism from $C(\overline{\mathcal{K}})$ into $C(\overline{\mathcal{K}})$, but it is not a surjective one since $\varphi_{0}\left(x_{0}\right)=\varphi_{0}(\infty)$. We also see that $T_{0}$ is a 2-local automorphism.

Theorem 2.3. Suppose that $F=\left\{f_{\alpha}\right\}$ is a (finite or infinite) subset of $C(\overline{\mathcal{K}})$ and the cardinality of $F$ is at most $\kappa$. Then there is an automorphism $T$ on $C(\overline{\mathcal{K}})$ such that $T_{0}\left(f_{\alpha}\right)=T\left(f_{\alpha}\right)$ for every $f_{\alpha} \in F$. In particular, $T_{0}$ is a 2-local automorphism. Thus the group of all automorphisms on $C(\overline{\mathcal{K}})$ is not algebraically reflexive.

Proof. Let $f$ be a function in $C(\overline{\mathcal{K}})$. Put

$$
\mathcal{M}_{f}=\{x \in \mathcal{L}: f(\infty) \neq f(x)\} .
$$

By a simple calculation we see that $\mathcal{M}_{f}$ is at most countable. It follows that the cardinality of $\cup_{f \in F} \mathcal{M}_{f}$ is at most $\kappa$. Put $\mathcal{M}=\mathcal{L} \backslash \cup_{f \in F} \mathcal{M}_{f}$. Since $\mathcal{L}=\mathcal{K} \backslash\left\{x_{n}\right\}_{n=0}^{\infty}$, the cardinality of $\mathcal{L}$ is $\nu$. Thus the cardinality of $\mathcal{M}$ equals to $\nu$ since $\nu$ is strictly larger than $\kappa$. In particular, $\mathcal{M}$ is an infinite set.

Let $\tau$ be a bijection form $\left\{x_{0}\right\} \cup \mathcal{M}$ onto $\mathcal{M}$. Define a homeomorphism $\varphi$ on $\overline{\mathcal{K}}$ as follows. For every $x \in\left\{x_{0}\right\} \cup \mathcal{M}$, put $\varphi(x)=\tau(x)$. Put $\varphi\left(x_{n}\right)=x_{n-1}$ for every $n \geq 1$ and $\varphi(y)=y$ for every $y \in \mathcal{L} \backslash \mathcal{M}$, and $\varphi(\infty)=\infty$. Then $\varphi$ is a homeomorphism since $\varphi(\infty)=\infty$. We show that $f \circ \varphi_{0}=f \circ \varphi$ for every $f \in F$. Since $\varphi_{0}(x)=\varphi(x)$ for every $x \in\left\{x_{n}\right\}_{n=1}^{\infty} \cup(\mathcal{L} \backslash \mathcal{M}) \cup\{\infty\}$, $f \circ \varphi_{0}=f \circ \varphi$ on this set. Since $\varphi\left(x_{0}\right)=\tau\left(x_{0}\right)$ is in $\mathcal{M}$ and $\varphi_{0}\left(x_{0}\right)=\infty$, we see that $f \circ \varphi\left(x_{0}\right)=f(\infty)=f \circ \varphi_{0}\left(x_{0}\right)$. Let $y \in \mathcal{M}$. Then $\varphi(y)=\tau(y) \in \mathcal{M}$. So $f \circ \varphi(y)=f(\infty)$. On the other hand, $\varphi_{0}(y)=y$ since $\mathcal{M} \subset \mathcal{L}$. Thus $f \circ \varphi_{0}(y)=f(y)=f(\infty)$ for $y \in \mathcal{M}$. So, $f \circ \varphi_{0}(y)=f \circ \varphi(y)$ holds for every $y \in \mathcal{M}$. It follows that $f \circ \varphi_{0}=f \circ \varphi$ on $\overline{\mathcal{K}}$. Put $T(h)=h \circ \varphi$ for $h \in C(\overline{\mathcal{K}})$. Then $T$ is an automorphism on $C(\overline{\mathcal{K}})$ and $T_{0}(f)=T(f)$ holds for every $f \in F$. Considering the case where $F$ consists of two functions, we see that $T_{0}$ is a 2-local automorphism. Since $T_{0}$ is not surjective, we see that $C(\overline{\mathcal{K}})$ is not algebraically reflexive by Theorem 2.2 .

It is natural to ask the following question.

Problem 2.4. If a unifrom algebra $A$ has the first countable maximal ideal space, are the groups of all automorphisms of $A$ and of all surjective isometries of $A$ always algebraically reflexive? 
Molnár and Zalar [13, Theorem 2.2] proved the case where $A=C(X)$. The authors do not know the answer in general.

\section{2-LOCAL ISOMETRIES}

Györy [4] proved that if $Y$ is a locally compact Hausdorff space which is first countable and $\sigma$-compact, then every 2-local isometry of $C_{0}(Y)$ is a surjective linear isometry, where $C_{0}(Y)$ denotes the algebra of all complex-valued continuous functions which vanish at infinity on $Y$. In this section we study 2-local isometries on uniform algebras, in particular, we show that 2-local isometries (resp. 2-local automorphisms) on uniform algebras on certain compact subsets of complex Euclidean spaces of dimension one and two are surjective linear isometries (resp. automorphisms).

In the case where $T$ is a surjective 2-local isometry on a uniform algebra, we see that $T$ is in fact a surjective linear isometry by applying the Mazur-Ulam theorem [9].

Theorem 3.1. Let $A$ be a uniform algebra on a compact Hausdorff space $X$. Suppose that $T$ is a surjective 2-local isometry. Then $T$ is a surjective linear isometry.

Proof. Let $f$ and $g$ be in $A$ and $\lambda \in \mathbb{C}$. Then there is a surjective linear isometry $T_{\lambda f, f}$ on $A$ with

$$
T(f)=T_{\lambda f, f}(f), \quad T(\lambda f)=\lambda T_{\lambda f, f}(f) .
$$

Thus we see that $T(\lambda f)=\lambda T(f)$. Furthermore there is a surjective linear isometry $T_{f, g}$ on $A$ such that $T_{f, g}(f)=T(f)$ and $T_{f, g}(g)=T(g)$. Thus by a theorem of de Leeuw, Rudin and Wermer [3, Theorem 3] there are function $h_{f, g} \in A$ with $\left|h_{f, g}\right|=1$ on $X$ and an automorphism $S_{f, g}$ on $A$ such that $T_{f, g}=h_{f, g} S_{f, g}$. Thus we have that

$$
\begin{aligned}
& \|T(f)-T(g)\|_{\infty(X)}=\left\|T_{f, g}(f)-T_{f, g}(g)\right\|_{\infty(X)} \\
& \quad=\left\|h_{f, g} S_{f, g}(f)-h_{f, g} S_{f, g}(g)\right\|_{\infty(X)}=\left\|S_{f, g}(f-g)\right\|_{\infty(X)} \\
& =\|f-g\|_{\infty(X)}
\end{aligned}
$$

Since $T$ is a surjection, we can apply the Mazur-Ulam theorem to get $T\left(\frac{f+g}{2}\right)=$ $\frac{T(f)+T(g)}{2}$, so $T(f+g)=T(f)+T(g)$ holds for every $f, g \in A$. It follows that $T$ is a surjective linear isometry on $A$.

Unless $T$ is assumed to be surjective, the Mazur-Ulam theorem cannot be applied (cf. [1]). We show that under a certain condition 2-local isometries on uniform algebras are isometrical isomorphisms. Every 2-local automorphisms on a uniform algebra is a 2-local isometry since an automorphism on a uniform algebra is a surjective linear isometry. We show a partial converse. 
Lemma 3.2. Let $A$ be a uniform algebra. Suppose that for every invertible function $h \in A$ with the constant modulus reduces to a constant function. Suppose that $T$ is a 2-local isometry on $A$ such that $T(1)=1$. Then $T$ is a 2-local automorphism.

Proof. For every $f \in A$, applying the 2-local-isometry condition for $T$ with $f$ and 1 , there exists a surjective linear isometry $T_{f, 1}$ on $A$ such that $T(f)=$ $T_{f, 1}(f)$ and $T(1)=T_{f, 1}(1)$. By a theorem of de Leeuw, Rudin and Wermer $\left[3\right.$, Theorem 3] we see that $T_{f, 1}$ is an automorphism since $T(1)=1$. Since the spectrum $\sigma\left(T_{f, 1}(f)\right)$ of $T_{f, 1}(f)$ coincides with that $\sigma(f)$ of $f$, we have $\sigma(T(f))=\sigma(f)$. Using this we see that $T(f+c)=T(f)+c$ holds for every pair $f \in A$ and a complex number $c$. In fact, by a theorem of de Leeuw, Rudin and Wermer [3, Theorem 3] and the hypothesis on $A$, there exist a complex number $h_{f+c, f}$ with the unit modulus and an automorphism $T_{f+c, f}$ with

$$
\begin{gathered}
T(f+c)=h_{f+c, f} T_{f+c, f}(f+c)=h_{f+c, f} T_{f+c, f}(f)+h_{f+c, f} c, \\
T(f)=h_{f+c, f} T_{f+c, f}(f) .
\end{gathered}
$$

Thus we have $T(f+c)=T(f)+h_{f+c, f} c$ and

$$
\begin{aligned}
\sigma(f)+c=\sigma(f+c)=\sigma(T(f+c)) & =\sigma\left(T(f)+h_{f+c, f} c\right) \\
& =\sigma(T(f))+h_{f+c, f} c=\sigma(f)+h_{f+c, f} c .
\end{aligned}
$$

We show that $h_{f+c, f}=1$ if $c \neq 0$. Let $a_{1} \in \sigma(f)$. Then by an induction there is a sequence $\left\{a_{n}\right\}$ in $\sigma(f)$ such that

$$
a_{n+1}=a_{n}+\left(1-h_{f+c, f}\right) c
$$

since $\sigma(f)+c=\sigma(f)+h_{f+c, f} c$. It follows that

$$
a_{n}=a_{1}+(n-1)\left(1-h_{f+c, f}\right) c .
$$

Since $\sigma(f)$ is a bounded set and $c \neq 0$, we see that $h_{f+c, f}=1$. Thus we see that $T(f+c)=T(f)+c$. Let $f$ and $g$ be functions in $A$. Put a complex number with $\sigma(f+c) \subset\{z \in \mathbb{C}: \operatorname{Re} z>0, \operatorname{Im} z>0\}$. As usual, we have

$$
T(f+c)=h_{f+c, g} T_{f+c, g}(f+c), \quad T(g)=h_{f+c, g} T_{f+c, g}(g)
$$

for a complex number $h_{f+c, g}$ with the unit modulus and an automorphism $T_{f+c, g}$. Since

$$
h_{f+c, g} \sigma\left(T_{f+c, g}(f+c)\right)=\sigma(T(f+c))=\sigma(f+c)=\sigma\left(T_{f+c, g}(f+c)\right)
$$

is in the $\{z \in \mathbb{C}: \operatorname{Re} z>0, \operatorname{Im} z>0\}, h_{f+c, g}$ must be 1 . It follows that $T(f)=T_{f+c, g}(f)$ and $T(g)=T_{f+c, g}(g)$. Thus we have that $\widetilde{T}$ is a 2-local automorphism. 
Theorem 3.3. Let $A$ be a uniform algebra. Suppose that for every invertible function $h \in A$ with the constant modulus reduces to a constant function. Then for any 2-local isometry $T$ on $A$, we have that $T(1)$ is a complex number with the unit modulus and that $\frac{T}{T(1)}$ is a 2-local automorphism on $A$, and an isometrical isomorphism from $A$ onto $T(A)$.

Proof. For every pair $f$ and $g$ in $A$, there exist a complex number $h_{f, g}$ with the unit modulus and an automorphism $T_{f, g}$ on $A$ such that $T(f)=h_{f, g} T_{f, g}(f)$ holds by a theorem of de Leeuw, Rudin and Wermer [3, Theorem 3] and the hypothesis on $A$. In particular, $T(1)=h_{1,0} T_{1,0}(1)=h_{1,0}$ follows that $T(1)$ is a complex number with the unit modulus. Put $\widetilde{T}=\frac{T}{T(1)}$. Then $\widetilde{T}$ is a 2-local isometry with $\widetilde{T}(1)=1$. Thus we see by Lemma 3.2 that $\widetilde{T}$ is a 2-local automorphism on $A$. Then by Theorem 2.2, $\widetilde{T}$ is an isometrical isomorphism from $A$ onto $T(A)$.

Let $K$ be a compact subset of $\mathbb{C}^{n}$. We denote by $A(K)$ the algebra of all complex-valued continuous functions on $K$ which are holomorphic on the interior Int $K$ of $K$. Sánchez and Molnár [18, p. 423] showed that the isometry group and the automorphism group of $A(K)$ are algebraically reflexive if $K$ is a compact subset of the complex plane $\mathbb{C}$ such that $\mathbb{C} \backslash K$ has finitely many components. We consider the case where $\mathbb{C} \backslash K$ has infinitely many components.

Theorem 3.4. Let $K \subset \mathbb{C}$ be a connected compact subset such that $\operatorname{Int} K$ has finitely many components and $K=\overline{\operatorname{Int} K}$, where $\overline{\operatorname{Int} K}$ is the closure of the interior of $K$. Then the group of all surjective linear isometries on $A(K)$ and the group of all automorphisms on $A(K)$ are algebraically reflexive.

Proof. Let $T$ be a locally surjective isometry. Then by [18, Theorem 5] there is a $\tau \in A$ with $\sigma(\tau) \subset \mathbb{T}$ and a unital endomorphism $\widetilde{T}$ such that $T=\tau \widetilde{T}$. Since $\tau$ is analytic on $\operatorname{Int} K$ and $\sigma(\tau) \subset \mathbb{T}$, we have by the maximum modulus principle that $\tau$ is a complex number with the unit modulus on each component of Int $K$. Since $K=\overline{\operatorname{Int} K}$ and $K$ is connected, we see that $\tau$ is a complex number with the unit modulus on $K$. Since $T$ is a locally surjective isometry, we see that $\widetilde{T}$ is also a locally surjective isometry on $A(K)$.

We show that $\widetilde{T}$ is in fact a local automorphism. Let $f \in A(K)$. Put a complex number $c$ with $\sigma(f+c) \subset\{z \in \mathbb{C}: \operatorname{Re} z>0, \operatorname{Im} z>0\}$. Since $\widetilde{T}$ is a locally surjective isometry, there exists a surjective linear isometry $\widetilde{T}_{f+c}$ on $A(K)$ with

$$
\widetilde{T}(f+c)=\widetilde{T}_{f+c}(f+c) .
$$

Applying [3, Theorem 3] we see that there are a complex number $a_{f+c}$ and an automorphism $S_{f+c}$ with

$$
\widetilde{T}(f+c)=a_{f+c} S_{f+c}(f+c) .
$$


Since $S_{f+c}$ is an automorphism, we have

$$
\sigma(\widetilde{T}(f+c))=a_{f+c} \sigma(f)+a_{f} c .
$$

On the other hand we have

$$
\sigma(\widetilde{T}(f+c))=\sigma(\widetilde{T}(f))+c \subset \sigma(f)+c
$$

since $\widetilde{T}$ is a unital endomorphism. It follows that

$$
a_{f+c} \sigma(f)+a_{f+c} c \subset \sigma(f)+c
$$

from which we have $a_{f+c}=1$ since $\sigma(f+c) \subset\{z \in \mathbb{C}: \operatorname{Re} z>0, \operatorname{Im} z>0\}$. We see that $\widetilde{T}(f+c)=S_{f+c}(f+c)$, so $\widetilde{T}(f)=S_{f+c}(f) ; \widetilde{T}$ is a local automorphism. Let id be the identitiy function on $\mathbb{C}$. Then there is an automorphism $\widetilde{T}_{\text {id }}$ such that $\widetilde{T}(\mathrm{id})=\widetilde{T}_{\text {id }}(\mathrm{id})$. Since the maximal ideal space of $A(K)$ is $K$ itself $[2$, Theorerm 3.5.7], and $\widetilde{T}$ and $\widetilde{T}_{\text {id }}$ are both unital, there are continuous functions $\varphi$ and $\psi$ from $K$ into $K$ such that $\widetilde{T}(g)=g \circ \varphi$ and $\widetilde{T}_{\mathrm{id}}(g)=g \circ \psi(g \in A(K))$. In particular, $\psi$ is a homeomorphism on $K$ and analytic on $\operatorname{Int} K$ since $\widetilde{T}_{\text {id }}$ is an automorphism. Since $\widetilde{T}(\mathrm{id})=\mathrm{id} \circ \varphi=\varphi$ and $\widetilde{T}_{\mathrm{id}}(\mathrm{id})=\mathrm{id} \circ \psi=\psi$, we have that $\varphi=\psi$. Thus we see that $\widetilde{T}=\widetilde{T}_{\mathrm{id}}$, that is, $\widetilde{T}$ is an automorphism. It follows that $T=\tau \widetilde{T}$ is a surjective linear isometry. We see that the group of all surjective linear isometries is algebraically reflexive. Then by Lemma 2.1 we also see that the group of all automorphisms is algebraically reflexive.

Corollary 3.5. Let $K \subset \mathbb{C}$ be a compact subset such that $\operatorname{Int} K$ is connected and $\overline{\operatorname{Int} K}=K$. Then the group of all surjective linear isometries on $A(K)$ and the group of all automorphisms on $A(K)$ are algebraically reflexive.

Since $K$ in the Corollary satisfies the hypotheses of Theorem 3.4, Corollary 3.5 holds.

We consider 2-local isometries and 2-local automorphisms on $A(K)$.

Theorem 3.6. Let $K$ be a connected compact subset of $\mathbb{C}$ such that Int $K$ has finitely many components and $\overline{\operatorname{Int} K}=K$. Suppose that $T$ is a 2-local isometry (resp. 2-local automorphism) on $A(K)$. Then $T$ is a surjective linear isometry (resp. automorphism).

Proof. Suppose that $h$ is a function in $A(K)$ with $\frac{1}{h} \in A(K)$ and $|h|=1$ on $K$. Then in the same way as in the proof of Theorem 3.4 we see that $h$ is a constant function. Thus $A(K)$ satisfies the condition in Theorem 3.3. Then by Theorem 3.3 we have that $T$ is linear. Then by the very definition, $T$ is a locally surjective isometry (resp. local automorphism). Thus by Theorem 3.4 we see that $T$ is a surjective isometry (resp. automorphism).

Let $K$ be a compact subset of $\mathbb{C}^{2}$. We denote by $P(K)$ the algebra of all complex valued continuous functions on $K$ which are uniformly approximated on $K$ by holomorphic polynomials. 
Theorem 3.7. Let $K$ be a connected compact subset of $\mathbb{C}^{2}$ such that $\operatorname{Int} K$ has finitely many components and $\overline{\operatorname{Int} K}=K$. Suppose that $T$ is a 2-local isometry (resp. 2-local automorphism) on $P(K)$. Then $T$ is a surjective linear isometry (resp. automorphism).

Proof. In the same way as before we see that $P(K)$ satisfies the conditions for $A$ in Theorem 3.3. Suppose that $T$ is a 2-local isometry (resp. 2-local automorphism). Then by Theorem 3.3 we see that $T(1)$ is a complex number with the unit modulus and $\widetilde{T}=\frac{T}{T(1)}$ is a 2-local automorphism. Note that $T(1)=1$ if $T$ is a 2-local automorphism. Thus there is an automorphism $S$ on $P(K)$ such that

$$
\widetilde{T}\left(\operatorname{id}_{1}\right)=S\left(\operatorname{id}_{1}\right), \quad \widetilde{T}\left(\operatorname{id}_{2}\right)=S\left(\operatorname{id}_{2}\right),
$$

where $\mathrm{id}_{j}$ is the function on $K$ defined by $\operatorname{id}_{j}\left(w_{1}, w_{2}\right)=w_{j}$ for every $\left(w_{1}, w_{2}\right) \in$ $K$. Thus we see that $S^{-1} \circ \widetilde{T}(P)=P$ holds for every holomorphic polynomial $P$ since $\widetilde{T}$ is an endomorphism by Theorem 3.3. It follows that $S^{-1} \circ \widetilde{T}(f)=f$ holds for every $f \in P(K)$. We conclude that $T=T(1) S$ and $T$ is a surjective linear isometry (resp. automorphism).

Theorem 3.8. Let $K$ be a closure of strictly pseudoconvex domain in $\mathbb{C}^{2}$ with the boundary of class $C^{2}$. Suppose that $T$ is a 2-local isometry (resp. 2local automorphism) on $A(K)$. Then $T$ is a surjective linear isometry (resp. automorphism).

Proof. As before we see that $A(K)$ satisfies the conditions for $A$ in Theorem 3.3. In a way similar to the proof of Theorem 3.7 there is an automorphism $S$ on $A(K)$ with

$$
S^{-1} \circ \widetilde{T}\left(\mathrm{id}_{1}\right)=\mathrm{id}_{1}, \quad S^{-1} \circ \widetilde{T}\left(\mathrm{id}_{2}\right)=\mathrm{id}_{2} .
$$

Let $f \in A(K)$ and $w=\left(w_{1}, w_{2}\right) \in \operatorname{Int} K$. Then by the Gleason property for $A(K)[15]$ there are functions $g, h \in A(K)$ such that

$$
f-f(w)=\left(\mathrm{id}_{1}-w_{1}\right) g+\left(\mathrm{id}_{2}-w_{2}\right) h .
$$

Since $S^{-1} \circ \widetilde{T}$ is a unital endmorphism on $A(K)$ we have that

$$
S^{-1} \circ \widetilde{T}(f-f(w))=\left(\operatorname{id}_{1}-w_{1}\right) S^{-1} \circ \widetilde{T}(g)+\left(\operatorname{id}_{2}-w_{2}\right) S^{-1} \circ \widetilde{T}(h),
$$

so

$$
S^{-1} \circ \widetilde{T}(f)(w)=f(w)
$$

holds for every $f \in A(K)$ and $w \in \operatorname{Int} K$. Since $\operatorname{Int} K$ is dense in $K$ we see that $S^{-1} \circ \widetilde{T}$ is the identity operator. It follows that $T=T(1) S$ and $T$ is a surjective linear isometry (resp. automorphism since $T(1)=1$ ). 
Definition 3.9. Let $B$ be a Banach algebra and $T$ a self-map on $B$. Suppose that $n$ is a positive integer. We say that $T$ is an $n$-local isometry (resp. $n$ local automorphism) if for every $n$-fold elements $a_{1}, a_{2}, \ldots, a_{n}$ of $B$ there exists a surjective linear isometry (resp. automorphism) $S$ on $B$ such that $T\left(a_{j}\right)=S\left(a_{j}\right)$ holds for every $1 \leq j \leq n$.

Note that $(n+1)$-local isometry (resp. $(n+1)$-local automorphism) is an $n$ local isometry (resp. $n$-local automorphism). Note also that a 3 -local isometry (resp. 3-local automorphism) $T$ on a Banach algebra $B$ is linear. (For $f, g \in B$ there is a surjective linear isometry $S$ on $B$ such that $T(f)=S(f), T(g)=$ $S(g)$ and $T(f+g)=S(f+g)$. Then $T(f+g)=T(f)+T(g)$ follows since $S(f+g)=S(f)+S(g)$. In a way similar, we see that $T(\lambda f)=\lambda T(f)(\lambda \in \mathbb{C}$, $f \in B)$.)

In a way similar to the proofs of Theorems 3.7 and 3.8, we see the following.

Theorem 3.10. Let $K$ be a connected compact subset of $\mathbb{C}^{n}$ such that $\operatorname{Int} K$ has finitely many components and $\overline{\operatorname{Int} K}=K$. Suppose that $T$ is an $n$-local isometry (resp. n-local automorphism) on $P(K)$. Then $T$ is a surjective linear isometry (resp. automorphism).

Theorem 3.11. Let $K$ be a closure of strictly pseudoconvex domain in $\mathbb{C}^{n}$ with the boundary of class $C^{2}$. Suppose that $T$ is an n-local isometry (resp. $n$-local automorphism) on $A(K)$. Then $T$ is a surjective linear isometry (resp. automorphism).

Problem 3.12. Is every 2-local isometry on a uniform algebra linear?

Problem 3.13. Is a 2-local isometry (resp. automorphism) a 3-local surjective isometry? In general, is an $n$-local surjective isometry a $(n+1)$-local surjective isometry?

An affirmative answer to Problem 3.13 follows by an affirmative answer to Problem 3.12.

\section{REFERENCES}

[1] J. A. Baker, Isometries in normed spaces, Amer. Math. Monthly 78(1971), 655-658

[2] A. Browder, "Introduction to Function Algebras", W.A. Benjamin 1969.

[3] K. de Leeuw, W. Rudin and J. Wermer, The isometries of some function spaces, Proc. Amer. Math. Soc. 11(1960), 694-698

[4] M. Győry, 2-local isometries of $C_{0}(X)$, Acta Sci. Math. (Szeged) 67(2001), 735-746

[5] K. Jarosz and T. S. S. R. K. Rao, Local isometries of function spaces, Math. Z. 243(2003), 449-469

[6] R. V. Kadison, Local derivations, Jour. Algebra 130(1990),494-509

[7] S. Kowalski and Z. Słodkowski, A characterization of multiplicative linear functionals in Banch algebras, Studia Math. 67((1980), 215-223

[8] D. R. Larson, Reflexivity, algebraic reflexivity and linear interpolation, Amer. Jour. Math. 110(1988), 283-299 
[9] S. Mazur and S. Ulam, Sur les transformations isométriques d'espaces vectoriels normes, C. R. Acad. Sci. Paris 194(1932), 946-948

[10] L. Molnár, 2-local isometries of some operator algebras, Proc. Edinburgh Math. 45(2002), 349-352

[11] L. Molnár, Some characterizations of the automorphisms of $B(H)$ and $C(X)$, Proc. Amer. Math. Soc. 130(2002), 111-120

[12] L. Molnár, Preserver problems on algebraic structures of linear operators and on function spaces, dissertation 2004

[13] L. Molnár and B. Zalar, Reflexivity of the group of surjective isometries on some Banach spaces, Proc. Edinburgh Math. Soc. 42(1999), 17-36

[14] W. P. Novinger, Linear isometries of subspaces of continuous functions, Studia Math. 53(1975), 273-276

[15] N. Øvrelid, Generators of the maximal ideals of $A(\bar{D})$, Pacifc Jour. Math. 39(1971), 219-223

[16] T. S. S. R. K. Rao, Local surjective isometries of function spaces, Expo. Math. 18(2000), 285-296

[17] F. C. Sánchez, The group of automorphisms of $L_{\infty}$ is algebraically reflexive, Studia Math. 16(2004), 19-32

[18] F. C. Sánchez and L. Molnár, Reflexivity of the isometry group of some classical spaces, Rev. Mat. Iberoamericana 18(2002), 409-430

[19] P. Šemrl, Local automorphisms and derivations on $\mathcal{B}(H)$, Proc. Amer. Math. Soc. 125(1997), 2677-2680

\section{Received: March 6, 2007}

